\title{
A Wireless ExG Interface for Patch-Type ECG Holter and EMG-Controlled Robot Hand
}

\author{
Kwangmuk Lee ${ }^{1}$, Yun Young Choi ${ }^{2}$, Dae Jung Kim ${ }^{2}$, Hee Young Chae ${ }^{1}$, Kyeonghwan Park ${ }^{1}$ (i), \\ Young Min $\mathrm{Oh}^{2}$, Sung Hun Woo ${ }^{2}$ and Jae Joon Kim ${ }^{2, *}$ \\ 1 School of Electrical and Computer Engineering, Ulsan National Institute of Science and Technology, Ulsan \\ 44919, Korea; leekm0226@unist.ac.kr (K.L.); laplume@unist.ac.kr (H.Y.C.); khpark@unist.ac.kr (K.P.) \\ 2 UMEDIX Co., Ltd., Seoul 06097, Korea; yychoi.choi@gmail.com (Y.Y.C.); djnara@gmail.com (D.J.K.); \\ oym.hazin@gmail.com (Y.M.O.); steven.shucom.woo@gmail.com (S.H.W.) \\ * Correspondence: jaejoon@unist.ac.kr; Tel.: +82-52-217-2126
}

Received: 19 July 2017; Accepted: 16 August 2017; Published: 16 August 2017

\begin{abstract}
This paper presents a wearable electrophysiological interface with enhanced immunity to motion artifacts. Anti-artifact schemes, including a patch-type modular structure and real-time automatic level adjustment, are proposed and verified in two wireless system prototypes of a patch-type electrocardiogram (ECG) module and an electromyogram (EMG)-based robot-hand controller. Their common ExG readout integrated circuit (ROIC), which is reconfigurable for multiple physiological interfaces, is designed and fabricated in a $0.18 \mu \mathrm{m}$ CMOS process. Moreover, analog pre-processing structures based on envelope detection are integrated with one another to mitigate signal processing burdens in the digital domain effectively.
\end{abstract}

Keywords: motion artifact; readout integrated circuit; ECG holter; robot-hand controller; envelope detector; level detector

\section{Introduction}

Electrophysiological monitoring devices such as electrocardiograms (ECGs) and electromyograms (EMGs) have inherent artifacts; that is, anomalous or interfering signals that originate from some sources other than the electrophysiological structure. Especially in wearable-type devices, which are preferred for 24-h monitoring systems and prosthesis control devices [1,2], the motion artifact is a crucial factor that dominates the overall monitoring quality, because most physiological devices generally operate in direct contact with human bodies [3,4]. Various physical movements give unstable skin contact, causing variations in the contact impedance and resulting in transient signal fluctuations. However, these motion artifacts are not easy to eliminate, because their frequency band overlaps with the frequency bands of electrophysiological signals, which degrades accuracy in the physiological signal analysis [5]. Therefore, there have been recent works to alleviate this motion-artifact problem by utilizing adaptive filtering [6], baseline wander tracking [7] or bio-impedance measurement [8]. However, the filtering works require additional detection channels for a reference, and baseline tracking requires a complex control loop that consists of an analog-to-digital converter (ADC), a digital-to-analog converter (DAC), and digital signal processing (DSP). The bio-impedance measurement is dedicated to heart rate, which is not appropriate for multi-sensor purposes. In terms of low-power DSP implementation, recent digital-domain efforts such as down-sampled wavelet transform [9], sub-Nyquist scanning [10], and data compression [11] have been reported to reduce the data rate from sensor modules or to relieve their post-processing and data-storage burdens. But, these digital efforts are still limited, because they do not fundamentally reduce the data rate of the sensors themselves. Meanwhile, one interesting analog pre-processing work on R-wave timing extraction from ECG measurement [12], whose digital implementation consumes three times more than its analog front end, has been reported. Even though 
it was implemented only for heart-rate detection, it is meaningful that analog pre-processing can reduce the burden of its digital-domain post-processing work. Therefore, conceptually inspired by this analog pre-processing method, we propose a reconfigurable ExG interface structure that is tolerant of various motion artifacts, and also reduces its post-processing burden in digital domain efficiently.

This work presents two wireless system prototypes of a patch-type ECG holter and an EMG-based robot-hand controller and their common ExG readout integrated circuit (ROIC) with multiple proposed circuit- and system-level technologies for motion-artifact reduction. In the case of the ECG detection, which has stringent requirements in terms of signal quality, its sensing module is made flexible by implementing each of its components on flexible principle printed circuit boards (PCBs); then, the whole module, including electrodes, is immobilized by putting adhesive patches on it. Through this patch-type implementation, a large portion of the motion artifacts are supposed to be autonomously removed. Then, in order to minimize the remaining artifacts, instant amplitude fluctuations are automatically compensated for by a proposed level detection scheme, and are also minimized by adopting state-of-the-art methods such as impedance boosting [13] and chopper stabilization [14].

For the EMG-based robot-hand control, the EMG module, whose signal-quality requirement is less strict, is designed in portable form for durability and reusability. Since the EMG signal has a higher frequency band, an envelope detection method is proposed to relieve its digital post-processing burden. Similarly, the R-peak timing information that is required for the ECG signal analysis in the digital domain is provided by a proposed simple analog method of a peak detection, resulting in significant reduction of its post-processing loads. For feasibility verification of these proposed methods, an ExG ROIC prototype and two system-level prototypes are fabricated and experimentally verified.

The remainder of this paper is organized as follows: Section 2 presents the proposed ExG interface architectures. Section 3 contains detailed designs of the ExG ROIC. Section 4 shows experimental results. Finally, the conclusion is given in Section 5.

\section{ExG Interface Architecture}

Figure 1 shows a proposed wireless ExG interface system to support the ECG monitoring and the EMG-based control, where wireless connectivity and battery are included together to minimize potential motion artifacts paths. The overall system is embodied as two wireless modules of a patch-type ECG holter and a portable-type EMG-based robot-hand, where a designed ExG ROIC is commonly utilized and their monitoring results are remotely displayed on a smartphone. For stable ambulatory ECG monitoring and effective reduction of motion artifact, a patch-type module is designed to implement most components on a flexible PCB that has polydimethylsiloxane (PDMS) substrate. One side of the module consists of the ExG ROIC, a microprocessor (MCU), a Bluetooth module, and two electrodes of the positive and the ground. The other side has a flexible lithium-ion battery and two electrodes of the negative and the ground. Through this hardware structure, two electrodes of the positive and the negative are autonomously located to have proper interspace around the heart for the ECG signal detection. For user convenience to control the robot hand intuitively, the EMG interface is designed to be a portable-type module which has similar composition of hardware components in the patch-type. The size of core PCB board is $5 \times 2.5 \mathrm{~cm}$ which is smaller than the ECG module and the rest area is filled with battery to give enough operation time. The EMG interface is configured to detect EMG signals from the arm muscles and to control the robot hand [15] wirelessly. 


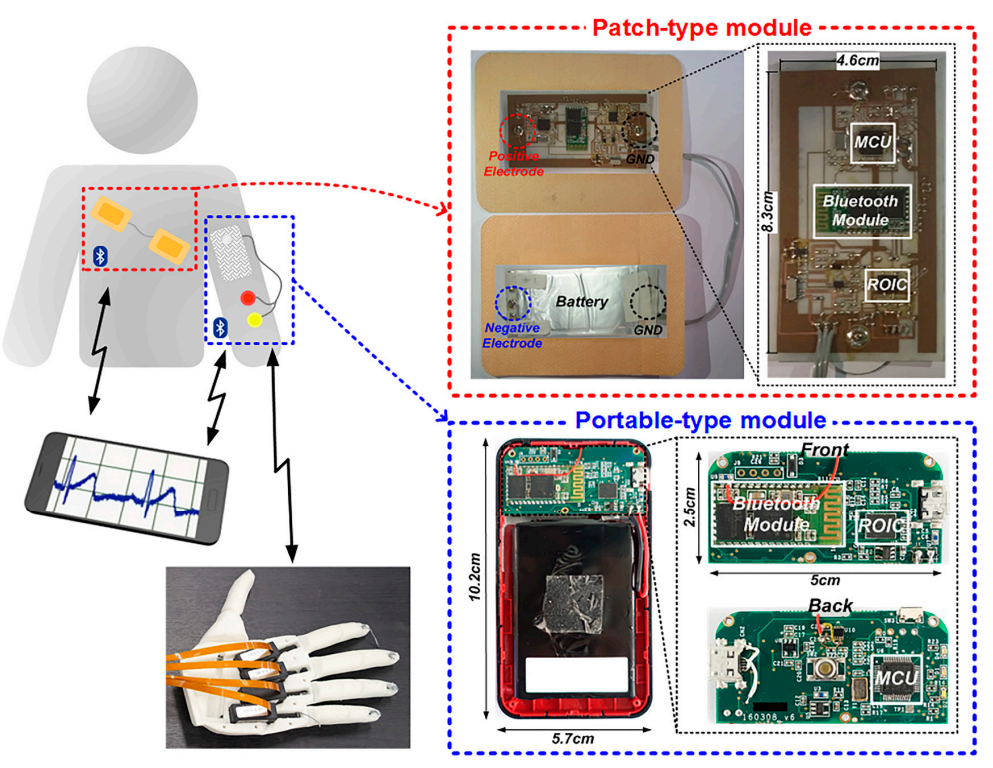

Figure 1. Wireless ExG interface with patch-type and portable-type modules for mobile healthcare and robot-hand control applications.

Figure 2 shows the architecture of the ExG ROIC that is commonly used in the two application systems. It consists of a capacitive-coupled instrumentation amplifier (CCIA), a programmable-gain amplifier (PGA), a level detector for real-time automatic gain calibration, and an envelope detector for amplitude detection of EMG signals. A low dropout (LDO) regulator, clock generators, bias circuits, a 12-bit successive approximation register (SAR) ADC [16], and a serial peripheral interface (SPI) are integrated together which includes a serial-to-parallel $(\mathrm{S} / \mathrm{P})$ register and parallel-to-serial $(\mathrm{P} / \mathrm{S})$ register. It is also designed to have reconfigurable structure to adjust voltage gain and frequency band depending on bio-potential signal types. In the ECG signal path, the PGA gain is automatically adjusted by a proposed level detector. The EMG path has another signal processing procedure of the envelope extraction to reduce its frequency bandwidth, which relieves its post-processing burden in digital domain. In this way, the proposed ROIC provides reconfigurable ExG interface whose internal operation mode is software-controlled. Another benefit of this software-controlled reconfigurable ROIC is to provide excellent protection capability from the reverse engineering of integrated circuits or systems because the hardware operation cannot be understood without its software information.

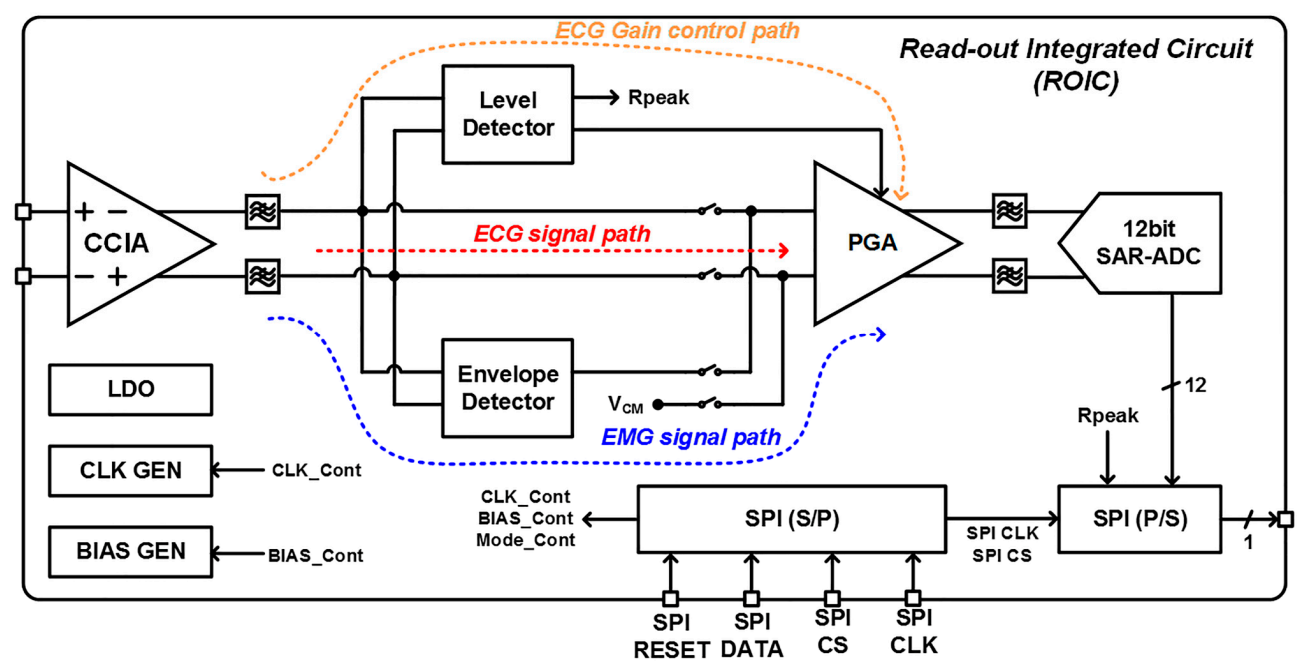

Figure 2. Block diagram of ExG readout integrated circuit (ROIC) for ECG/EMG interfaces. 


\section{ROIC Implementation}

\subsection{CCIA and PGA}

The schematic of the CCIA, which is a front-end low-noise amplifier to remove low-frequency noise and DC offsets, is shown in Figure 3a, where it employs capacitive feedback to set mid-band gain of $\mathrm{C}_{\mathrm{in} 1} / \mathrm{C}_{\mathrm{fb} 1}$. The input capacitor $\mathrm{C}_{\mathrm{in} 1}$ and the feedback capacitor $\mathrm{C}_{\mathrm{fb} 1}$ are designed to be $5 \mathrm{pF}$ and $250 \mathrm{fF}$, respectively, in order to realize the $26 \mathrm{~dB}$ mid-band gain. The chopper stabilization technique [14] is adopted to suppress low-frequency noises including $1 / \mathrm{f}$ noises. The input impedance is increased further to minimize remaining motion-artifact effects by adopting capacitive impedance boosting [13], which implements a positive feedback between input and output nodes without additional current consumption. The resulting input impedance is maximized when $C_{i b l}$ is almost equal to $C_{f b 1}$. In order to achieve a low high-pass cutoff frequency for DC-offset reduction, the feedback resistor $R_{f b 1}$ is implemented with a PMOS pseudo-resistor that achieves very high resistance in small area [17].

The schematic of PGA in the second stage, which is a kind of switched-capacitor amplifier, is shown in Figure $3 b$, where the variable resistors $R_{\mathrm{in} 2}$ and $R_{\mathrm{fb} 2}$ are implemented by capacitors of $C_{\mathrm{sc} \_ \text {in } 2}$ and $\mathrm{C}_{\mathrm{sc} \_ \text {fb2 }}$, and switches with non-overlapping clocks of CLKP and CLKN. The PGA is designed to set mid-band gain $\left(\mathrm{C}_{\mathrm{sc} \_ \text {in2 }} / \mathrm{C}_{\mathrm{sc}_{-} \mathrm{fb} 2}\right)$, and its low-pass cutoff frequency is changed by adjusting the feedback capacitor $\mathrm{C}_{\mathrm{fb} 2}$ based on the operation modes of the ECG and the EMG. For sufficient noise immunity, fully-differential amplifiers (FDAs) in the PGA and the CCIA are commonly designed to have folded-cascode structures with rail-to-rail input ranges. While the FDA for the CCIA, including its common-mode feedback circuits, is optimized to minimize the power consumption, the FDA for the PGA is designed to have current-driving capability for output loads.

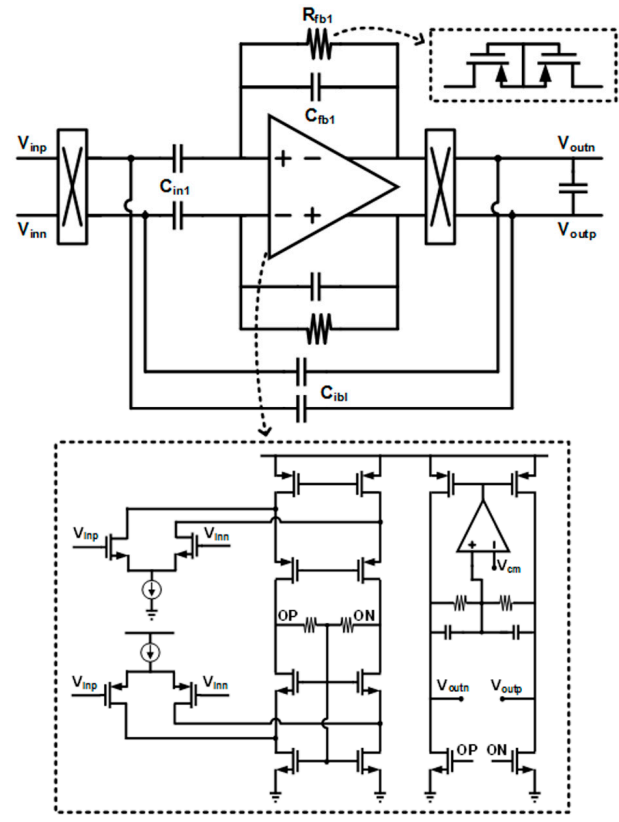

(a)

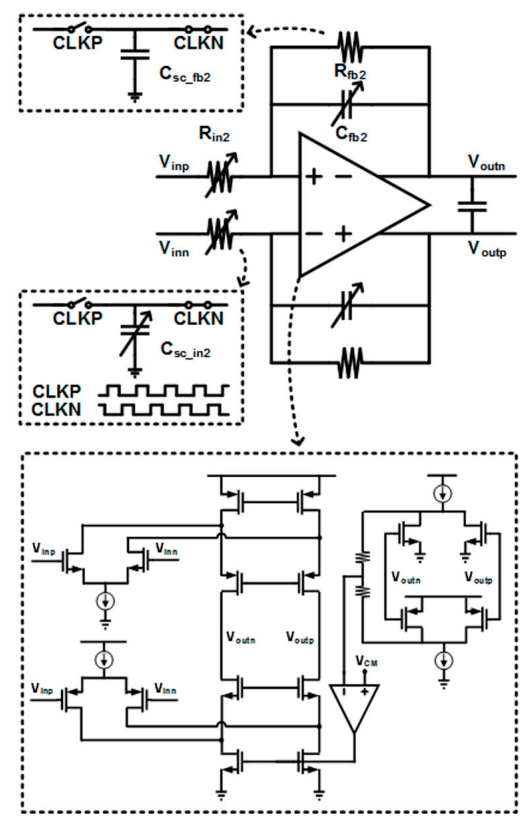

(b)

Figure 3. Schematics of (a) CCIA and (b) PGA.

\subsection{Envelope and Level Detectors}

The robot-hand system is designed to be controlled by referring the EMG signal strength, where important control information exists in the amplitude of instantaneous EMG spikes. Therefore, the EMG signals are converted to low-frequency amplitude signals by utilizing the envelope detector, reducing their sampling rate in the process of digital conversion, and thereby reducing the digital post-processing burden considerably. The schematic and basic operation of the designed envelope 
detector, which is based on the peak detector in [18], are shown in Figure 4. If any input among $V_{\text {inp }}$ and $V_{\text {inn }}$ is greater than the output of $V_{\text {out }}$, the current mirror chain of $M_{5,6}$ and $M_{7,8}$ provides current proportional to their difference, and the peak value of the inputs is stored in the output through an external capacitor $\mathrm{C}_{\mathrm{ext}}$. For continuous tracking of time-varying input signals, the output voltage is slowly discharged by utilizing a leakage current $\mathrm{I}_{\mathrm{L}}$, which comes from three diode-connected transistors. Since the size ratio of $\mathrm{M}_{1,2}$ and $\mathrm{M}_{3,4}$ affects the feedback amount, the output DC offset can be adjusted by changing the $\mathrm{M}_{3,4}$ size.
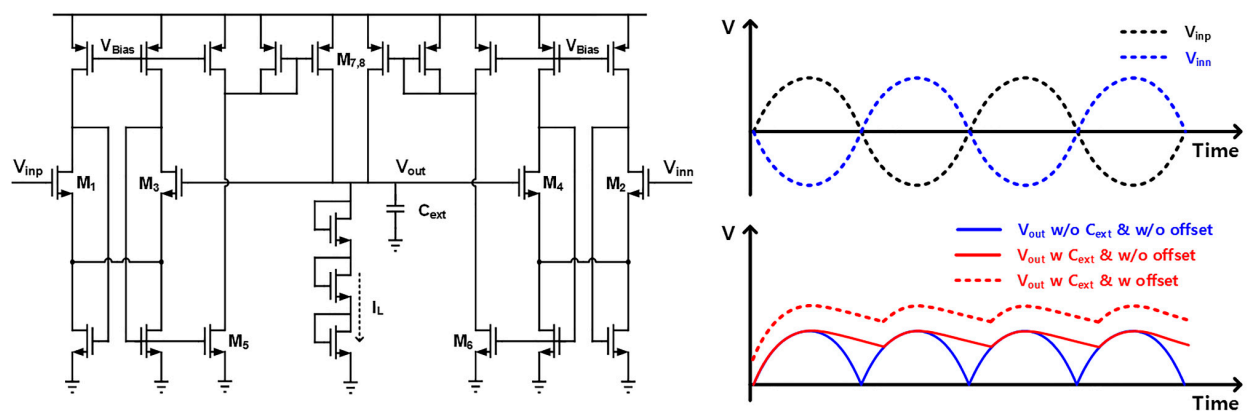

Figure 4. Schematic and operational principle of the envelope detector.

Figure 5 shows the architecture of the proposed ECG level detector and the operational principle of its automatic gain control on the PGA with detection capability of the R-peak signal. The envelope detector is used to find the R-peak in analog domain to reduce the post-processing burden that finds the R-peak location of the ECG signal in digital domain, where it is designed to have some output offset-voltage control range by adjusting the size of $\mathrm{M}_{3,4}$. The input signal of the level detector, which is processed to $\mathrm{V}_{\text {pre, }}$ comes from a differential output of CCIA, which is a first-stage amplifier in the ROIC. The envelope detector generates the ECG baseline signal with some offset $\left(V_{\text {env }}\right)$, and it is compared with $\mathrm{V}_{\text {pre }}$ to produce the $\mathrm{R}$-peak location signal of $\mathrm{D}_{\mathrm{R} \text {-peak }}$. If the $\mathrm{D}_{\mathrm{R} \text {-peak }}$ becomes too high, it activates a peak-to-digital converter (PDC), which is composed of a comparator, an 8-bit resistive digital-to-analog converter (DAC), and successive approximate register (SAR) control logic. The PDC output, which represents a digital value of the R-peak signal in the ECG, is converted to the PGA control signal of $D_{\text {cont. }}$ In this way, the ECG signal amplitude is automatically controlled to give stable output waveforms, making the overall system less susceptible to motion-artifact fluctuations. The sensitivity of this automatic amplitude control can be adaptively changed by tuning the programming of the mapping method from $\mathrm{D}_{\mathrm{R} \text {-peak }}$ to $\mathrm{D}_{\text {cont }}$, depending on operating environments.

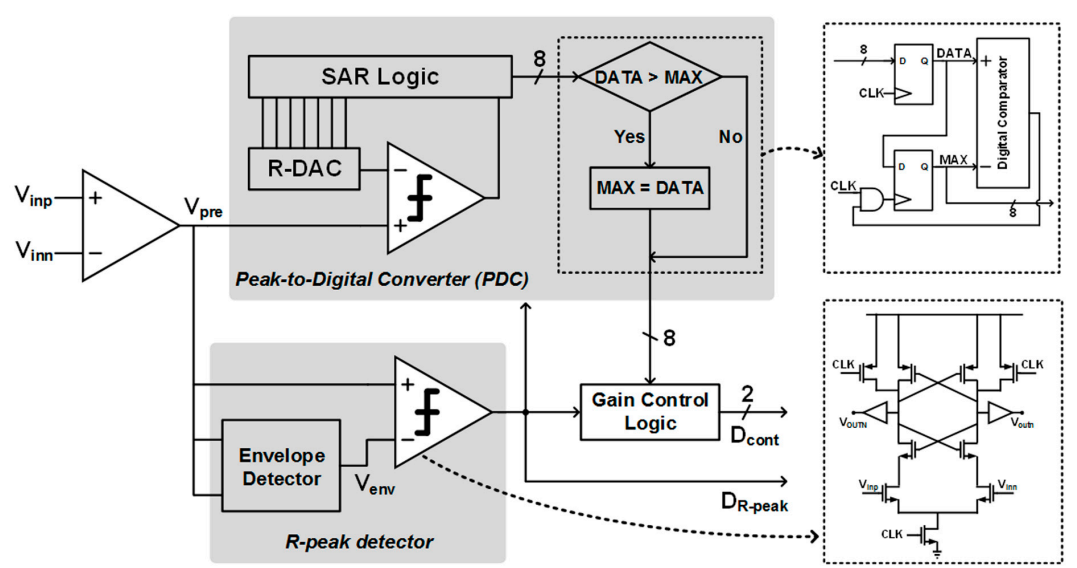

(a)

Figure 5. Cont. 


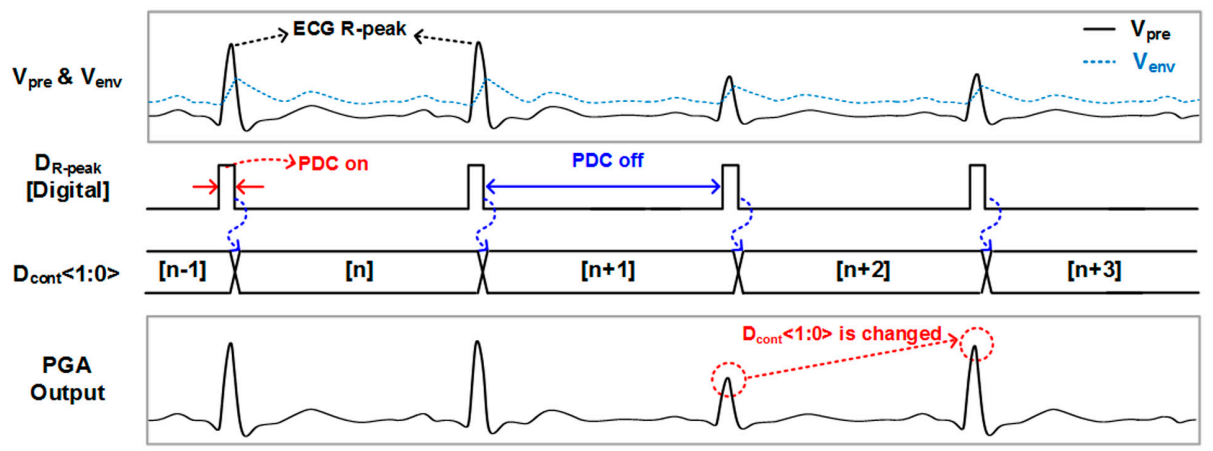

(b)

Figure 5. ECG level detector: (a) circuit diagram and (b) operation principle.

\section{Experimental Results}

A proposed ExG ROIC prototype was fabricated in a $0.18 \mu \mathrm{m}$ complementary metal oxide semiconductor (CMOS) process, and Figure 6 shows its microphotograph with a chip area of $4 \mathrm{~mm}^{2}$. The external supply voltage is $3 \mathrm{~V}$, but the LDO inside the ROIC provides an internal supply voltage of $1.8 \mathrm{~V}$ for better noise immunity and device protection. For verification of the ROIC prototype, the gain control capability was measured as shown in Figure 7a, where the low-pass corner frequency of the PGA was adjusted together. The measured pass band was set to be 0.6 to $230 \mathrm{~Hz}$ in the ECG mode and 0.6 to $1.5 \mathrm{kHz}$ in the EMG mode. The measured programmable pass-band gain range was 31.3 to $44.8 \mathrm{~dB}$, and the power consumption was $37.3 \mu \mathrm{W}$. At $60 \mathrm{~Hz}$, the common-mode rejection ratio (CMRR) was $65.6 \mathrm{~dB}$ and power-supply rejection ratio (PSRR) was $55.4 \mathrm{~dB}$. Figure $7 \mathrm{~b}$ shows measured characteristic of the SAR ADC, where the spurious-free dynamic range (SFDR) is $67.38 \mathrm{~dB}$ and the power consumption is $0.2 \mu \mathrm{W}$ at $125 \mathrm{~S} / \mathrm{s}$ sampling rate.

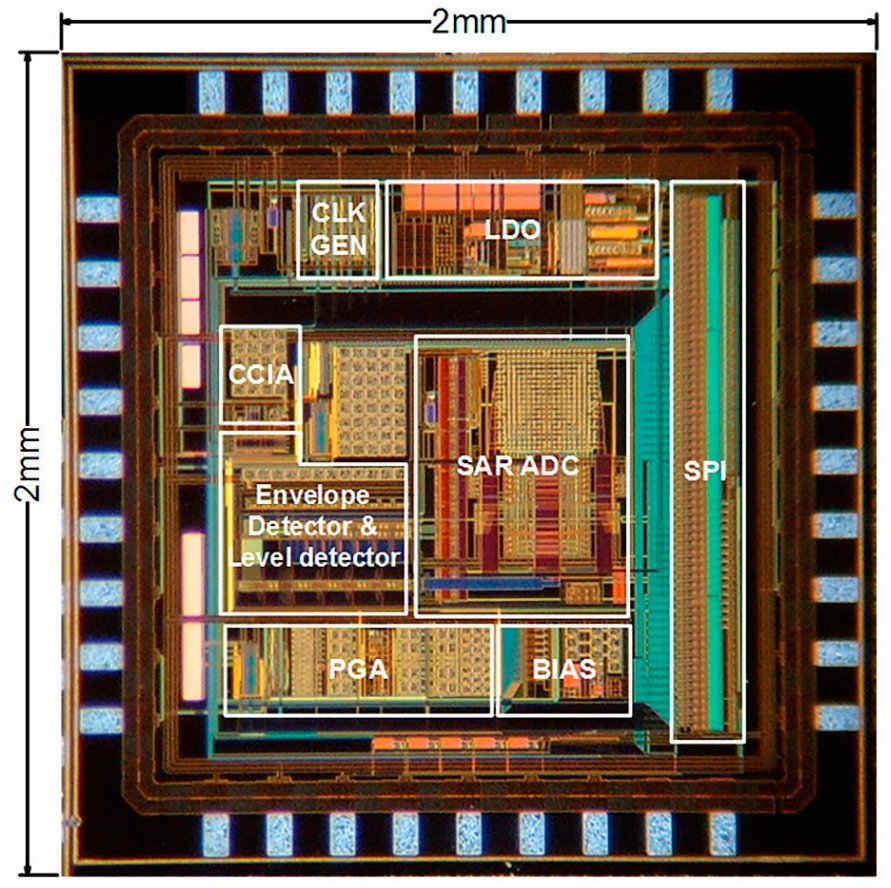

Figure 6. Microphograph of ExG ROIC prototype. 


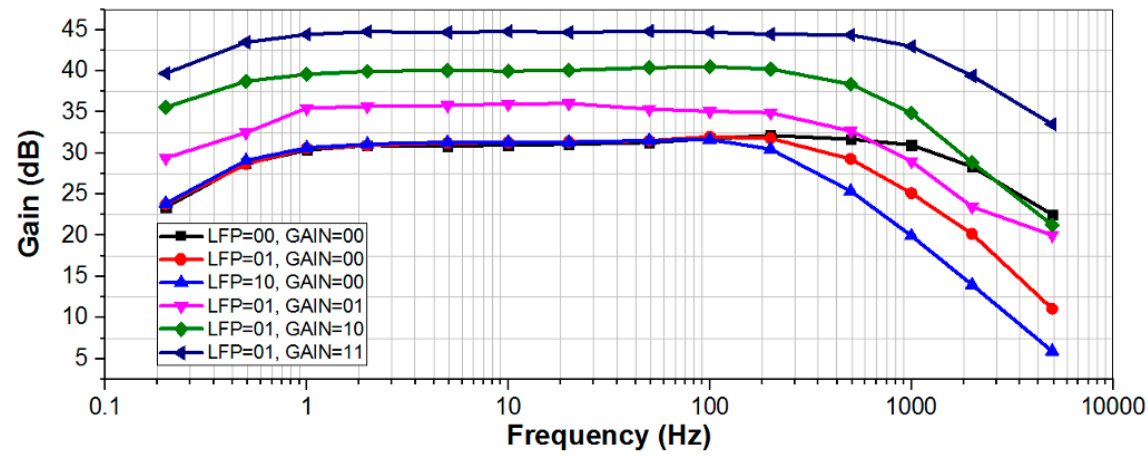

(a)

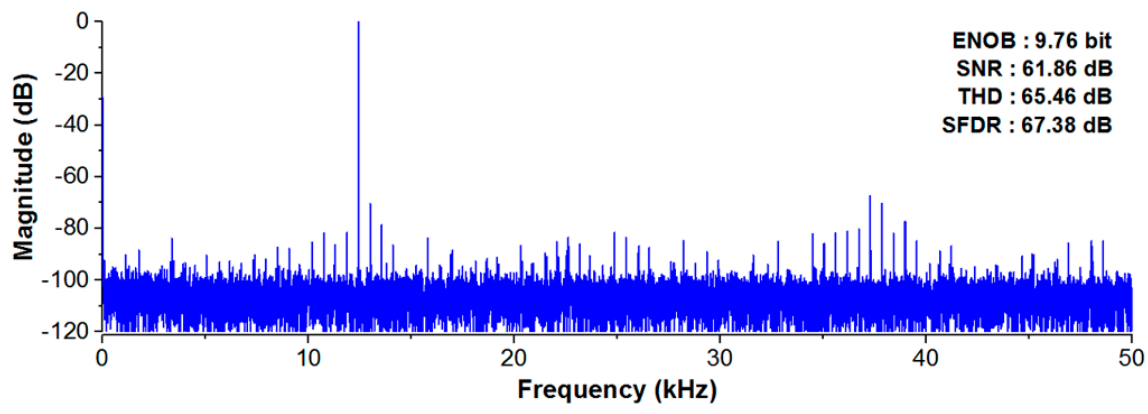

(b)

Figure 7. Measured characteristics of (a) ExG ROIC; and (b) SAR ADC.

By utilizing the fabricated ExG ROIC, two system-level prototypes of the patch type and the portable type were manufactured as seen in Figure 1. First, the ECG module that consists of the ROIC, electrodes (3 M Ag/AgCl), the MCU (ARM cortex-M0), the Bluetooth (HC-06), and the flexible battery was implemented as patch-type to immobilize every component for anti-artifacts. For feasibility verifications of the proposed motion-artifact reduction methods, the comparison experiment of the wireless ECG patch prototype against a commercial ECG holter product (TLC5000 of Contec Medical Systems) was performed as shown in Figure 8, where electrodes are attached according to the standard 12-lead ECG placement [5]. The commercial holter's waveform corrupted seriously when the human moved up and down (in moving state), while the proposed prototype gave stable waveforms in both stationary and moving states, and the P-QRS-T wave was clearly observed. In the proposed prototype, the R-peak waveform, which is generated from the proposed level detector, was displayed together, which would initiate its digital post-processing sequences to analyze the ECG waveform characteristics. Through this comparison experiment, it can be seen that the proposed anti-artifact methods are more attractive in the form of wearable devices because the adhesive patch increases the anti-artifact capability of the ROIC.

Figure 9a shows the experimental environment of EMG-based wireless robot-hand control interface, which is composed of the proposed portable-type EMG module and a robot-hand controller. The robot-hand controller consists of the MCU, the Bluetooth, a DC-motor driver, and a commercial robot hand of Open Bionics, which includes one micro linear actuator at each finger. During this experiment, the EMG envelope waveform depending hand movement is shown in Figure 9b. The EMG envelope signal shows low-frequency amplitude waveforms, while the original EMG signal gives high-frequency AC waveforms. The robot-hand control interface for mimicking hand activity is easily implemented by utilizing the EMG envelope signal, rather than the original. In Figure $9 c, d$, it can been seen that the robot fingers moved following the human finger movement intuitively. Through this experiment, the effectiveness of the envelope-based EMG interface to simplify its post-processing was verified, where intuitive control capability of the robot hand was achieved through the EMG-based mimic mechanism. 


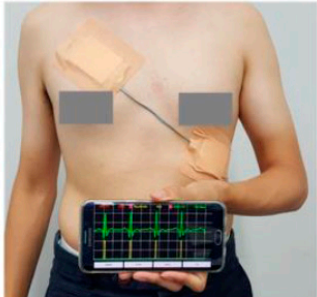

Proposed ECG module

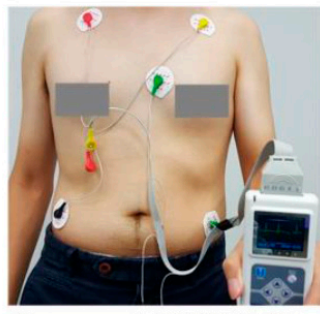

Commercial ECG holter

(a)
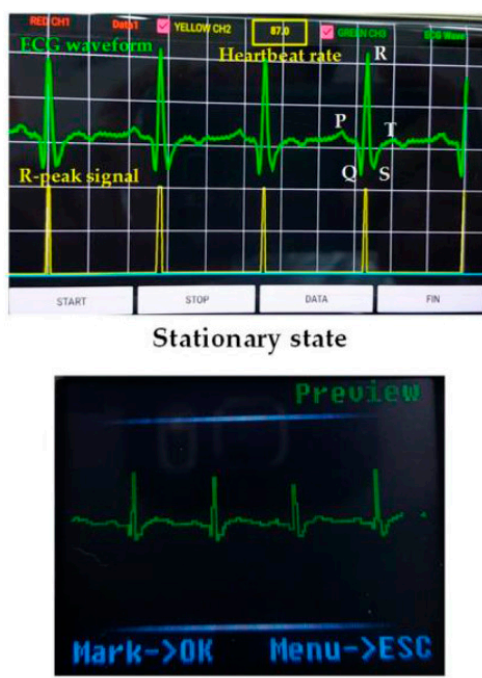

Stationary state

(b)
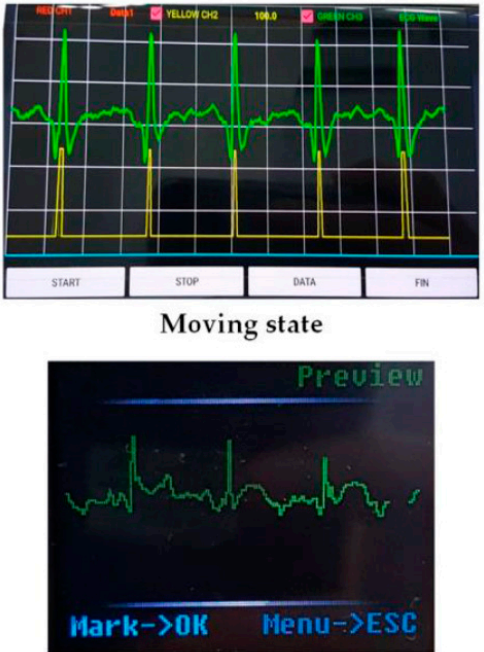

Moving state

(c)

Figure 8. Comparison of experimental results of the proposed ECG prototype versus commercial ECG holter (TLC5000 of Contec Medical Systems). (a) Experiment environment; (b) Comparison results in stationary state; (c) Comparison results in moving state.

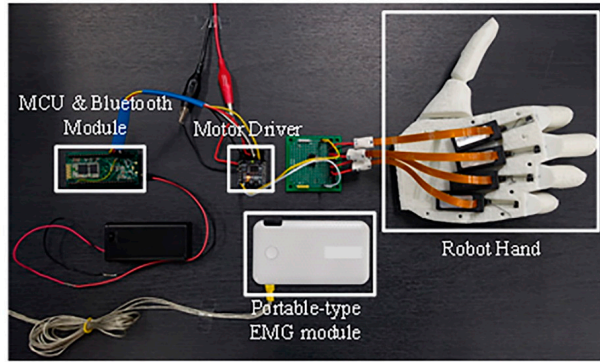

(a)

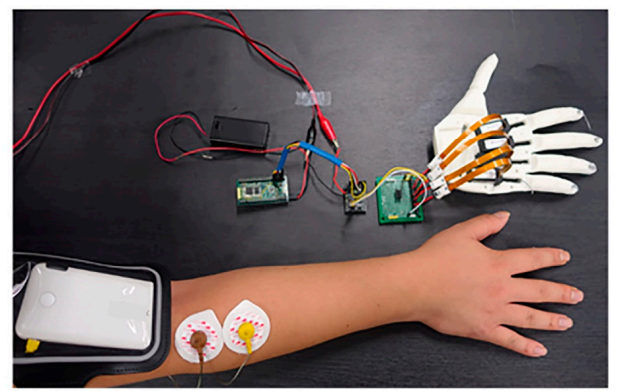

(c)

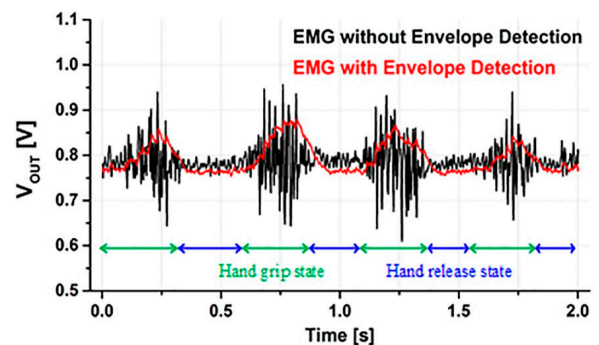

(b)

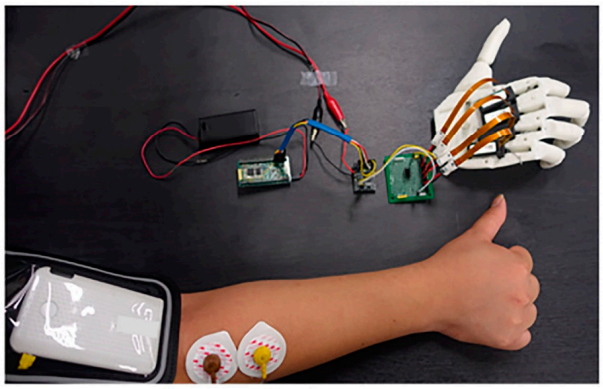

(d)

Figure 9. Experiment of robot-hand controller interface. (a) Experiment environment; (b) EMG waveform with and without envelope detection; (c) Robot-hand control in hand release state; (d) Robot-hand control in hand grip state.

In this way, the designed common ExG interface was experimentally verified to support both the ECG and the EMG, which provides a one-channel sensor interface. Measured performances are summarized in Table 1, where comparisons with recent works are also given. The designed ROIC provides multiple electrophysiological interfaces through the proposed reconfigurable structure, embedding distinguished features of anti-artifact and pre-processing functions. For further applications, including standard 12-lead ECG recording [5] and multi-point EMG detection of various 
muscle movements [15], the common ROIC would be redesigned to support multi-channel ExG interfaces, where Bluetooth might need to be replaced with WiFi in order to cover multiple streams of data traffic from multiple channels.

Table 1. Performance summary and comparison with recent works.

\begin{tabular}{|c|c|c|c|c|}
\hline Parameter & This Work & [7] & [12] & [13] \\
\hline ROIC Application & ECG, EMG, EEG & ECG & ECG & ECG, EEG \\
\hline Module type & $\begin{array}{l}\text { Patch-type ECG module, } \\
\text { Portable-type EMG module }\end{array}$ & N.A. & $\begin{array}{l}\text { Ear clip-type ECG } \\
\text { module }\end{array}$ & N.A \\
\hline Functionality & $\begin{array}{c}\text { R-peak detection, } \\
\text { EMG envelope detection }\end{array}$ & $\begin{array}{l}\text { Baseline wander } \\
\text { tracking }\end{array}$ & R-peak detection & N.A. \\
\hline Process & $0.18 \mu \mathrm{m}$ CMOS & N.A. & $0.18 \mu \mathrm{m}$ CMOS & $0.18 \mu \mathrm{m}$ CMOS \\
\hline Chip Area $\left(\mathrm{mm}^{2}\right)$ & $4 \mathrm{~mm}^{2}$ & N.A. & $3.24 \mathrm{~mm}^{2}$ & $24.01 \mathrm{~mm}^{2}$ \\
\hline Passband (Hz) & $\begin{array}{c}0.6-1500 \mathrm{~Hz} \\
\text { (programmable) }\end{array}$ & DC-500 Hz & $0.5-22 \mathrm{~Hz}$ & $\begin{array}{c}1-100 \mathrm{~Hz} \\
\text { (programmable) }\end{array}$ \\
\hline Gain $(\mathrm{dB})$ & $\begin{array}{c}\text { 31.3-44.8 dB } \\
\text { (programmable) }\end{array}$ & $48 \mathrm{~dB}$ & $\begin{array}{c}47-88 \mathrm{~dB} \\
\text { (programmable) }\end{array}$ & $\begin{array}{c}47.3-71.9 \mathrm{~dB} \\
\text { (programmable) }\end{array}$ \\
\hline Supply voltage (V) & $1.8 \mathrm{~V}$ (ROIC) $3 \mathrm{~V}$ (Module) & $3 \mathrm{~V}$ & $0.8 \mathrm{~V}$ & $3.3 \mathrm{~V}$ \\
\hline $\begin{array}{c}\text { Power } \\
\text { consumption }(\mu \mathrm{W})\end{array}$ & $\begin{array}{l}37.3 \mu \mathrm{W} \text { (ROIC) } \\
0.2 \mu \mathrm{W}(\mathrm{ADC})\end{array}$ & $\begin{array}{c}\text { N.A. (ROIC) } \\
160 \mu \mathrm{W} \text { (ADC) }\end{array}$ & $58 \mathrm{nW}(\mathrm{ROIC})$ & $12.5 \mu \mathrm{W}$ (ROIC) \\
\hline $\begin{array}{l}\text { ADC resolution } \\
\text { (bit) }\end{array}$ & 12 bit & 12 bit & N.A. & 12 bit \\
\hline
\end{tabular}

\section{Conclusions}

A reconfigurable ExG interface structure that is immune to motion artifacts and reduce digital post-processing burden considerably was proposed and experimentally verified in the form of two wireless electrophysiological system prototypes. For ECG interface, the patch-type wireless sensing module was developed to include two proposed features of automatic level adjustment and R-peak timing pre-processing. The wireless robot-hand control system prototype, which is based on the EMG-envelope detection, was implemented to mimic the movement of the human hand. Inside both system prototypes, the CMOS-fabricated ROIC was included and verified to work as their common ExG readout interface.

Supplementary Materials: The following are available online at http:/ /www.mdpi.com/1424-8220/17/8/1888/s1, Video S1: Comparison experiment results of proposed ECG prototype and commercial ECG holter in stationary and moving states.

Acknowledgments: This work was supported by the Technology Innovation Program (10064058: Development of patch-type 7 modal multi-sensor devices and platform technology for wearable healthcare applications) funded by the Ministry of Trade, Industry and Energy, South Korea and also supported by the Basic Science Research Program through the National Research Foundation of Korea funded by the Ministry of Education under Grant 2016R1D1A1B03930404.

Author Contributions: Kwangmuk Lee, Yun Young Choi, Kyeonghwan Park, and Jae Joon Kim conceived and designed the multi-sensor interface architecture; Kwangmuk Lee, Yun Young Choi, Kyeonghwan Park, and Hee Young Chae implemented the ROIC and performed its system experiments; Dae Jung Kim, Hee Young Chae, Young Min Oh, and Sung Hun Woo implemented the two prototype modules and wireless systems. Kwangmuk Lee and Jae Joon Kim wrote the paper.

Conflicts of Interest: The authors declare no conflict of interest.

\section{References}

1. Konijnenburg, M.; Stanzione, S.; Yan, L.; Jee, D.-W.; Pettine, J.; van Wegberg, R.; Kim, H.; van Liempd, C.; Fish, R.; Schuessler, J.; et al. A Multi(bio)sensor Acquisition System with Integrated Processor, Power Management, $8 \times 8$ LED Drivers, and Simultaneously Synchronized ECG, BIO-Z, GSR, and Two PPG Readouts. IEEE J. Solid State Circuits 2016, 51, 2584-2595. [CrossRef] 
2. Woo, S.-H.; Choi, Y.-Y.; Kim, D.-J.; Bien, F.; Kim, J.-J. Tissue-Informative Mechanism for Wearable Non-invasive Continuous Blood Pressure Monitoring. Sci. Rep. 2014, 4. [CrossRef] [PubMed]

3. Yoo, H.-J.; van Hoof, C. Bio-Medical CMOS ICs, 1st ed.; Springer: New York, NY, USA, 2010; p. 32. ISBN 978-1-4419-6596-7.

4. Choi, S.; Kim, D.-J.; Choi, Y.-Y.; Park, K.; Kim, S.-W.; Woo, S.-H.; Kim, J.-J. A Multisensor Mobile Interface for Industrial Environment and Healthcare Monitoring. IEEE Trans. Ind. Electron. 2017, 64, 2344-2352. [CrossRef]

5. Gupta, R.; Mitra, M.; Bera, J. ECG Acquisition and Automated Remote Processing, 1st ed.; Springer: New York, NY, USA, 2014; p. 53. ISBN 978-81-322-1556-1.

6. Torfs, T.; Chen, Y.-H.; Kim, H.; Yazicioglu, R.-F. Noncontact ECG Recording System with Real Time Capacitance Measurement for Motion Artifact Reduction. IEEE Trans. Biomed. Circuits Syst. 2014, 8, 617-625. [CrossRef] [PubMed]

7. Niederhauser, T.; Marisa, T.; Kohler, L.; Haeberlin, A.; Wildhaber, R.-A.; Abächerli, R.; Goette, J.; Jacomet, M.; Vogel, R. A Baseline Wander Tracking System for Artifact Rejection in Long-Term Electrocardiography. IEEE Trans. Biomed. Circuits Syst. 2016, 10, 255-265. [CrossRef] [PubMed]

8. Lee, J.; Cho, S. A Motion-tolerant Heart Rate Detection Method Using Bio-impedance and MUSIC Algorithm. In Proceedings of the 2015 IEEE Sensors, Busan, Korea, 1-4 November 2015; pp. 1-4.

9. Liu, X.; Zhou, J.; Yang, Y.; Wand, B.; Lan, J.; Wang, C.; Luo, J.; Goh, W.-L.; Kim, T.T.-H.; Je, M. A 457 nW Near-Threshold Cognitive Multi-Functional ECG Processor for Long-Term Cardiac Monitoring. IEEE J. Solid State Circuits 2014, 49, 2422-2434. [CrossRef]

10. Moy, T.; Huang, L.; Rieutort-Louis, W.; Wu, C.; Cuff, P.; Wagner, S.; Sturm, J.C.; Verma, N. An EEG Acquisition and Biomarker-Extraction System Using Low-Noise-Amplifier and Compressive-Sensing Circuits Based on Flexible, Thin-Film Electronics. IEEE J. Solid State Circuits 2017, 52, 309-321. [CrossRef]

11. Deepu, C.-J.; Zhang, X.; Liew, W.-S.; Wong, D.L.T.; Lian, Y. An ECG-on-Chip with 535 nW/Channel Integrated Lossless Data Compressor for Wireless Sensors. IEEE J. Solid State Circuits 2014, 49, 2435-2448. [CrossRef]

12. He, D.-D.; Sodini, C.-G. A 58 nW ECG ASIC With Motion-Tolerant Heartbeat Timing Extraction for Wearable Cardiovascular Monitoring. IEEE Trans. Biomed. Circuits Syst. 2015, 9, 370-376. [CrossRef] [PubMed]

13. Song, H.; Park, Y.; Kim, H.; Ko, H. Fully Integrated Biopotential Acquisition Analog Front-End IC. Sensors 2015, 15, 25139-25156. [CrossRef] [PubMed]

14. Enz, C.-C.; Temes, G.-C. Circuit Techniques for Reducing the Effects of Op-Amp Imperfections: Autozeroing, Correlated Double Sampling, and Chopper Stabilization. Proc. IEEE 1996, 84, 1584-1614. [CrossRef]

15. Fuketa, H.; Yoshioka, K.; Shinozuka, Y.; Ishida, K.; Yokota, Y.; Matsuhisa, N.; Inoue, Y.; Sekino, M.; Sekitani, T.; Takamiya, M.; et al. $1 \mu \mathrm{m}$-Thickness Ultra-Flexible and High Electrode-Density Surface Electromyogram Measurement Sheet with 2 V Organic Transistors for Prosthetic Hand Control. IEEE Trans. Biomed. Circuits Syst. 2014, 8, 824-833. [CrossRef] [PubMed]

16. Park, K.; Kim, S.; Eom, W.; Kim, J.-J. A Reconfigurable Readout Integrated Circuit for Heterogeneous Display-based Multi-Sensor Systems. Sensors 2017, 17. [CrossRef] [PubMed]

17. Tu, C.-C.; Lin, T.-H. Measurement and parameter characterization of pseudo-resistor based CCIA for biomedical applications. In Proceedings of the IEEE International Symposium on Bioelectronics and Bioinformatics (IEEE ISBB), Chung Li, Taiwan, 11-14 April 2014; pp. 1-4.

18. Park, S.-B.; Wilson, J.-E.; Ismail, M. The CHIP-Peak Detectors for Multistandard Wireless Receivers. IEEE Circuits Devices Mag. 2006, 22, 6-9. [CrossRef]

(c) 2017 by the authors. Licensee MDPI, Basel, Switzerland. This article is an open access article distributed under the terms and conditions of the Creative Commons Attribution (CC BY) license (http://creativecommons.org/licenses/by/4.0/). 